\title{
Synthesis of Diethyl Malonate under the Catalysis of Expandable Graphite
}

\author{
Xiu-yan Pang*, Xiu-zhu Shi \\ College of Chemistry and Environmental Science, Hebei University, Baoding 071002, China \\ Email: pxy833@163.com
}

\begin{abstract}
In the synthesis of diethyl malonate with ethanol and malonic acid as reactants, the catalytic activity of expandable graphite (EG) was investigated, which was prepared by simple and rapid method with natural flake graphite, potassium permanganate and sulfuric acid (75\%) as per the mass ratio of 1.0:0.15:6.67. The ethanol to acid mole ratio, EG dosage and its repetitive effectiveness were determined through single factor experiments. Appropriate condition for the synthesis of diethyl malonate was determined as: ethanol to malonic acid mole ratio was 6.0:1.0, EG was added as per an $8 \%$ mass percentage of the total quality of reactants, cyclohexane was added at a level of $20 \mathrm{~mL}$, the reaction maintained slight boiling state until no water separated. Under the above condition, yield of diethyl malonate reached $77.6 \%$. Repetition experiments showed that the catalyst could be reused three times without obvious loss of activity. However, it was significantly reduced in the $4^{\text {th }}$ time. Nevertheless, its expansion performance almost remained unchanged.
\end{abstract}

Keywords: Sulfuric acid, expandable graphite, diethyl malonate, reutilization, catalytic activity.

\section{Introduction}

The diethyl malonate, which is slight soluble in water and soluble in organic solvent, shows important uses in the synthesis of carboxylic acids and their derivatives due to its active methylene group. As a raw material and intermediate ${ }^{[1,2]}$, diethyl malonate and its derivatives have been widely used in the preparation of polyester, coating, pharmacy and as additive of cosmetics. Therefore, its production is of great importance.

As for the chemical synthesis of diethyl malonate, the mainly reported means include sodium cyanide method, interesterification ${ }^{[3]}$, esterification, carbonylation. With regard to sodium cyanide method, the chemical reaction between mono chloroacetic acid and sodium cyanide is unfriendly to environment and it involves complicated procedures ${ }^{[4]}$. While, the palladium $\left(\operatorname{Pd}\left(\mathrm{Ph}_{3} \mathrm{P}\right)_{4}\right)$ catalyzed carbonylation of ethyl chloroacetate in the presence of phase transfer agent $\left(\mathrm{Bu}_{4} \mathrm{NI}\right)$ showed a high yield of $93 \%{ }^{[5]}$, but the high cost of $\mathrm{Pd}\left(\mathrm{Ph}_{3} \mathrm{P}\right)_{4}$ deserved consideration. As for the esterification between malonic acid and ethanol, $\mathrm{H}_{2} \mathrm{SO}_{4}$ may be the normally used catalyst due to its high catalytic activity. Whereas, this kind of catalyst is difficult to reclamine and has a series of problems such as serious corrosive effect for equipment, dehydration and carbonification effect for organic reactants and products. Therefore, the eco-friendly and easily recycled catalysts had been reported in the synthesis of diethyl malonate ${ }^{[6,7]}$.

Expandable graphite (EG) is a kind of graphite intercalation compound normally preprocessed with sulfuric acid ${ }^{[8]}$, and the formation of the graphite sulfate as showed in equation (1) turns it into a solid acid ${ }^{[9]}$. Therefore, EG has exhibited catalytic activity in the synthesis of ketones ${ }^{[10]}$ and esters ${ }^{[11]}$. The EG can be prepared with cheap material and at gentle temperature, and it's also easy to disperse or recycle. However, there is no report on its application to the synthesis of diethyl malonate. In this research, a type of EG was prepared with $\mathrm{KMnO}_{4}$ as oxidant and $\mathrm{H}_{2} \mathrm{SO}_{4}$ as intercalator, and its catalysis for esterification reaction between malonic acid and ethanol was investigated. The influence of malonic acid to ethanol mole ratio, EG dosage, reaction temperature and water-carrying reagent on diethyl malonate yield was tested. Catalytic activity differences between the $\mathrm{EG}$ and $\mathrm{H}_{2} \mathrm{SO}_{4}$, and differences between the EGs with different repetition times were investigated. 


$$
n(\text { graphite })+n \mathrm{H}_{2} \mathrm{SO}_{4}+\frac{n}{2}[\mathrm{O}] \rightarrow\left[\text { graphite } \cdot \mathrm{HSO}_{4}^{-} \cdot \mathrm{H}_{2} \mathrm{SO}_{4}\right]_{n}+\frac{n}{2} \mathrm{HO}
$$

$[\mathrm{O}]$ : oxidant; $\left[\right.$ graphite $\left.\cdot \mathrm{HSO}_{4^{-}} \cdot \mathrm{H}_{2} \mathrm{SO}_{4}\right]$ : the produced graphite sulfate

\section{Materials and Methods}

A 650 full-automatic refractmeter (Shandong, China), X-ray diffractometer (XRD) (Dandong, China), KSW Muffle furnace (Tianjin, China), FTS-40 Fourier transform infra-red (FTIR) spectrometer (America Biorad) were used in experiments.

Natural flake graphite with an average diameter of $0.30 \mathrm{~mm}$ and a carbon content of $96 \%$ was provided by Qingdao Xite Carbon Co. Ltd., China. Ethanol, malonic acid, cyclohexane, $\mathrm{H}_{2} \mathrm{SO}_{4}(98 \%)$, $\mathrm{KMnO}_{4}, \mathrm{CaCl}_{2}, \mathrm{Na}_{2} \mathrm{CO}_{3}$ were all analytical reagents.

\subsection{Preparation of the EG}

EG was prepared according to the reported method as per the mass ratio $\mathrm{C}: 75 \% \mathrm{H}_{2} \mathrm{SO}_{4}: \mathrm{KMnO}_{4}$ of 1.0:6.67:0.15 ${ }^{[12]}$. Graphite intercalation reaction totally lasted $30 \mathrm{~min}$ at $30^{\circ} \mathrm{C}$ controlled with a water bath. The obtained EG showed an initial expansion temperature (the temperature corresponding to the volume expanding to 1.5 times of the EG initial value) of $210^{\circ} \mathrm{C}$ and expanded volume of $520 \mathrm{~mL} / \mathrm{g}$ at $800^{\circ} \mathrm{C}$.

\subsection{X-Ray Diffraction (XRD) Spectra of Natural Graphite and the EG}

XRD data of the prepared EG and natural graphite were recorded from $15^{\circ}$ to $70^{\circ}$, and the interlayer spacing was obtained as per Bragg's law. As showed in Fig. 1, the EG presents the same characteristic diffraction peaks like natural graphite at about $26^{\circ}$ and $55^{\circ}$, which indicates EG still keeps the layer structures. Whereas, the (002) diffraction peak transfers to a smaller angle of $25.9^{\circ}$ and corresponds to a big interplanar crystal spacing of $3.44 \AA$. This is because that natural graphite is oxidized by $\mathrm{KMnO}_{4}$, and the gap between graphite layers is extended due to repulsion between positive charges, then intercalation reaction as showed in equation (1) proceeds between graphite and intercalator.

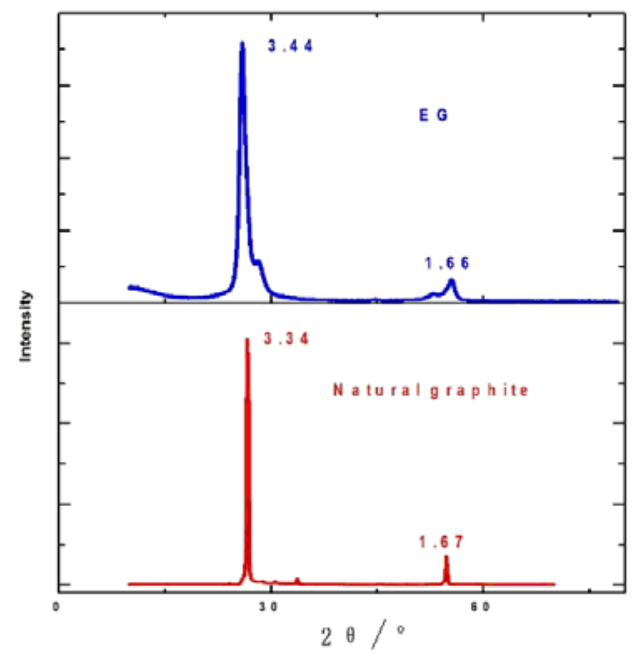

Figure 1. XRD spectra of graphite and the EG

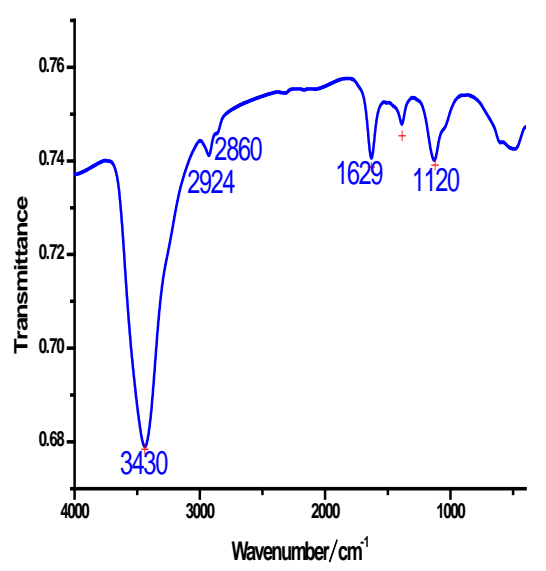

Figure 2. FTIR spectrum of the EG

\subsection{Fourier Transform Infrared (FTIR) Spectrum of the Prepared EG}

The FTIR spectrum of the prepared EG was recorded between 4000-400 $\mathrm{cm}^{-1}$. As can be seen from Fig. 2, the intercalation of $\mathrm{H}_{2} \mathrm{SO}_{4} / \mathrm{HSO}_{4}^{-}$causes the EG showing the characteristic stretching vibration 
absorption peaks of $-\mathrm{OH}$ at $3400 \mathrm{~cm}^{-1}{ }^{[8]}$. The symmetry and dissymmetry stretching vibration absorption of $\mathrm{CH}_{2}$ are observed in the range of $2930-2850 \mathrm{~cm}^{-1}$, which should be the results of a part of $\mathrm{C}=\mathrm{C}$ being damaged. The peak at about $1630 \mathrm{~cm}^{-1}$ is the specific absorption of the $\mathrm{C}=\mathrm{C}$ stretching vibration, originating from the graphite conjugated structure. The strong stretching vibration absorption peak of sulphate is also observed round $1120 \mathrm{~cm}^{-1}$.

\subsection{Preparation of Diethyl Malonate}

With a definite mole ratio, malonic acid, ethanol, water-carrying reagent and catalyst were added into the three-necked bottle appended with thermometer, magnetic stirrer, water segregator and reflux condenser. The reaction was kept under a slight boiling state until no water evaporated from the reactor. Cooled to room temperature and filtrated under vacuum, then the collected filtrate was distilled at air pressure until the temperature climbed to $100^{\circ} \mathrm{C}$. The distilled residue was cooled and severally washed with $\mathrm{NaHCO}_{3}$ saturated solution and deionized water. After desiccation with $\mathrm{CaCl}_{2}$, the collected oily matter was analyzed with refractmeter and FTIR respectively.

In air pressure distillation process, the $199.3^{\circ} \mathrm{C}$ (boiling point of diethyl malonate) is not used for controlling temperature for the following reasons: (1) Diethyl malonate is easy to decompose and produce malonic acid at $170^{\circ} \mathrm{C}$; (2) In the reaction system, the possible existing substances such as water, ethanol, water-carrying reagent possess lower boiling point than $100^{\circ} \mathrm{C}$; (3) The residual malonic acid can be thoroughly removed with $\mathrm{NaHCO}_{3}$ saturated solution, deionized water and $\mathrm{CaCl}_{2}$. Therefore, the distillation can be carried out at air pressure and a temperature lower than $100^{\circ} \mathrm{C}$.

\subsection{Characterization of the Diethyl Malonate}

Refractive index of the collected oily matter is detected as $\mathrm{n}_{\mathrm{d}}{ }^{20}=1.4134$, and it's similar with the reported value of 1.4135 for diethyl malonate.

The FTIR spectrum of the collected oily matter was detected and showed in Fig. 3. Except the -OH, the characteristic peaks of $\mathrm{C}-\mathrm{H}\left(2986 \mathrm{~cm}^{-1}\right), \mathrm{C}=\mathrm{O}\left(1739 \mathrm{~cm}^{-1}\right)$ and $\mathrm{O}=\mathrm{C}-\mathrm{O}\left(1163 \mathrm{~cm}^{-1}\right)$ are all observed. The result is similar with the reported standard spectrum of diethyl malonate, and therefore, the collected oily matter is the wanted diethyl malonate.

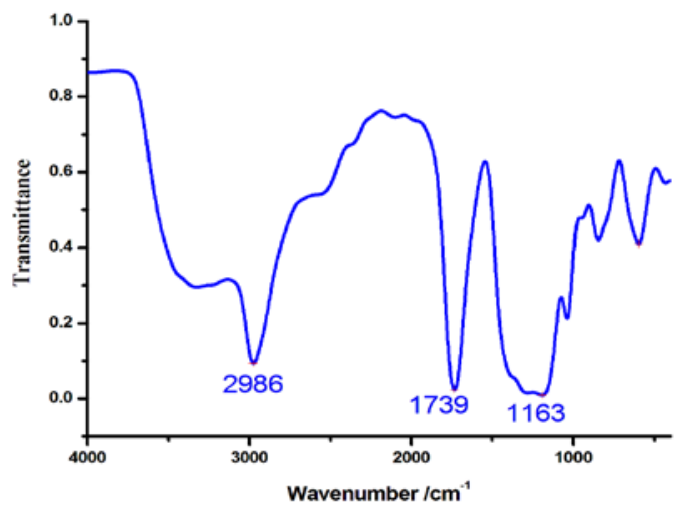

Figure 3. FTIR spectrum of the collected sample

In all the tested experiments, diethyl malonate yield was calculated according to equation (2).

$$
\text { Yield } \%=\left(m_{\text {diethyl malonateobtained }} / m_{\text {diethyl malonatetheoritical calculated }}\right) * 100 \%
$$




\section{$3 \quad$ Results and Discussion}

\subsection{Influence of Ethanol to Malonic Acid Mole Ratio on Ester Yield}

To find the feasible mole ratio of ethanol to malonic acid, a series of experiments were implemented with the reaction maintaining at a slight boiling state until no water separated, with volume of cyclohexane at $20 \mathrm{~mL}$ and EG at $8 \mathrm{wt} \%$ of the total malonic acid and ethanol mass respectively. As showed in Table 1, the yield increases with the increasing ethanol to acid mole ratio in the tested range of 3.0:1-7.0:1, but the growth rate becomes very slight when it's above 6.0:1. Although the diethyl malonate yield mainly depends on the concentration of ethanol, too high ethanol dosage would obviously debase the relative concentration of malonic acid. So ethanol to acid mole ratio of 6.0:1.0 is adopted in the subsequent experiments.

Table 1. Influence of ethanol to malonic acid mole ratio on diethyl malonate yield.

\begin{tabular}{llllll}
\hline nethanol : nmalonic acid & $3.0: 1$ & $4.0: 1$ & $5.0: 1$ & $6.0: 1$ & $7.0: 1$ \\
Diethyl malonate yield /\% & 22.4 & 32.3 & 44.8 & 77.6 & 78.9 \\
\hline
\end{tabular}

\subsection{Influence of EG Quantity on Ester Yield}

With the reaction maintaining at a slight boiling state, mole ratio of ethanol to malonic acid at 6.0:1 and volume of cyclohexane at $20 \mathrm{~mL}$, influence of the EG quantity on diethyl malonate yield was detected. As tested in single factor experiments, diethyl malonate yield increases with the increasing EG dose in the range of 7.0-8.0 wt\% as showed in Table 2, and then it gets smaller and smaller along with the increasing catalyst quantity, which should be caused by the adsorption of EG for diethyl malonate. Therefore, the relative mass percentage of EG can be set as $8 \mathrm{wt} \%$ of the total ethanol and acid mass.

Table 2. Influence of EG quantity on diethyl malonate yield

\begin{tabular}{llllll}
\hline Dose of EG /\% & 7.0 & 7.5 & 8.0 & 8.5 & 9.0 \\
Diethyl malonate yield/\% & 58.3 & 74.2 & 77.6 & 72.3 & 64.9 \\
\hline
\end{tabular}

\subsection{Influence of Reaction Temperature on Ester Yield}

The relationship between thermo dynamitic parameters $\Delta \mathrm{rH}_{\mathrm{m}}{ }^{\ominus}, \Delta \mathrm{rS}_{\mathrm{m}}{ }^{\ominus}, \Delta \mathrm{rG}_{\mathrm{m}}{ }^{\ominus}$ of diethyl malonate and temperature is showed as equation $(3),(4),(5)^{[13]}$, and the results indicate that low temperature is in favor of the improvement of reaction equilibrium constant, and separation of the produced water is beneficial for the formation of diethyl malonate.

$$
\begin{gathered}
\Delta r H_{m}{ }^{\theta}=-40978.2+138.5 T+0.0695 T^{2}-0.6067 \times 10^{-3} T^{3} \\
\Delta r S_{m}{ }^{\theta}=-714.1+138.5 \ln T+0.139 T-0.910 \times 10^{-3} T^{2} \\
\Delta r G_{m}{ }^{\theta}=-40978.2+852.6 T-138.5 T \ln T-0.0695 T^{2}+0.303 \times 10^{-3} T^{3}
\end{gathered}
$$

The synthesis of diethyl malonate is carried out in three-necked bottle appended with water segregator, reflux condenser, and cyclohexane is tested as a low temperature water-carrying reagent. To insure the separation of produced water from reactor, the lowest reaction temperature should be kept above $68.95^{\circ} \mathrm{C}$ (boiling point of cyclohexane-water azeotrope). The inactivation temperature of the EG is $210^{\circ} \mathrm{C}$ (the initial expansion temperature of the EG as mentioned above). In theory, the reaction temperature can be controlled in the range of $68.95-210^{\circ} \mathrm{C}$. In fact, when cyclohexane is used as watercarrying reagent, boiling point of the reaction system can reach only $72^{\circ} \mathrm{C}$. Therefore, it is favor of the improvement of diethyl malonate yield when the reaction maintains slight boiling state. 


\subsection{Influence of Water-Carrying Reagent and Water Segregator on Ester Yield}

Water-carrying reagent contributes to the release of water, and then impels the reaction to occur in the direction of producing ester. The selected water-carrying reagent should be able to form azeotrope with a low boiling point, which is not only in favor of the formation of ester, but also reduce the heat consumption. The boiling point of cyclohexane-water azeotrope is $68.95^{\circ} \mathrm{C}$. With the reaction maintaining at a slight boiling state, mole ratio of ethanol to acid at 6.0:1, and the mass percentage of EG at $8 \%$, the influence of cyclohexane and reflux condenser on diethyl malonate yield was tested. As showed in Table 3, diethyl malonate yield can reach $66.2 \%$ with no water-carrying reagent and water segregator. On the contrary, when water segregato is installed, the reaction gets a low yield of $58.9 \%$ due to the distilling out of ethanol. It achieves the highest value of $77.6 \%$ with the coexistence of cyclohexane and water segregator. Therefore, the concentration of ethanol and water is the same important for the improvement of diethyl malonate yield. $20 \mathrm{~mL}$ cyclohexane is used in the reaction.

Table 3. Influence of cyclohexane and water segregator on ester yield

\begin{tabular}{ccc}
\hline Cyclohexane & Water segregator & Diethyl malonate yield $/ \%$ \\
\hline no & no & 66.2 \\
no & yes & 58.9 \\
yes & yes & 77.6 \\
\hline
\end{tabular}

\subsection{Feasible Condition of Preparing Diethyl Malonate with EG as Catalyst}

According to the above mentioned experiments, the feasible conditions to get diethyl malonate are described as follows: mole ratio between ethanol and malonic acid is $6.0: 1$, the relative quantity of EG is $8 \mathrm{wt} \%$ and cyclohexane is added at a level of $20 \mathrm{~mL}$. Diethyl malonate final yield can reach $77.6 \%$ within about $1.0 \mathrm{~h}$ with the reaction maintaining at slight boiling state.

\subsection{Comparison of Catalytic Activity}

To investigate catalytic activity difference between the EG and $\mathrm{H}_{2} \mathrm{SO}_{4}$ with a weight concentration of $98 \%$, and difference between the EGs with different repetition times, parallel and contrast experiments were carried out under the mentioned reaction conditions. Results listed in Table 4 show that EG presents higher catalytic activity than concentrated $\mathrm{H}_{2} \mathrm{SO}_{4}$, which is because the former can effectively control the dehydration and carbonification effect for organic reactants and products. The recycling experiments of the EG indicate that this catalyst possesses high stability. After reused for three times, the ester yield can still reach $60.4 \%$. But it suddenly decreases to $26.3 \%$ when the EG repeats for the $4^{\text {th }}$ time. It had been testified that the leaching of intercalator such as $\mathrm{H}_{2} \mathrm{SO}_{4}$ would be weaken or prohibited when the EG was further treated with water or $\mathrm{H}_{2} \mathrm{SO}_{4}$ solution at high temperature ${ }^{[14]}$. It's because the treatment in liquid and high temperature condition causes the change of EG edge structure, which increases the mass transfer resistance between solid phase and liquor phase, and then leads to a lower catalytic activity in the $4^{\text {th }}$ time. The change of edge structure is irreversible, thus EG cannot be further recycled as catalyst. It is worthy to note that expanded volume of the EG has no obvious change, it still maintains $510 \mathrm{~mL} / \mathrm{g}$. So esterification reaction does not change EG's expandable capacity and it can be further used as adsorbent or flame retardant ${ }^{[15,16]}$.

Table 4. The catalytic activity difference between different catalysts

\begin{tabular}{lllccc}
\hline Catalysts & $\begin{array}{l}\mathrm{H}_{2} \mathrm{SO}_{4} \\
/(98 \%)\end{array}$ & $\begin{array}{l}\text { EG for the } 1^{\text {st }} \\
\text { time }\end{array}$ & $\begin{array}{l}\text { EG for the } 2^{\text {nd }} \\
\text { time }\end{array}$ & $\begin{array}{l}\text { EG for the } 3^{\text {rd }} \\
\text { time }\end{array}$ & $\begin{array}{l}\text { EG for the } 4^{\text {th }} \\
\text { time }\end{array}$ \\
\hline $\begin{array}{l}\text { Diethyl malonate } \\
\text { yield } / \%\end{array}$ & 60.0 & 77.6 & 70.6 & 60.4 & 26.3 \\
\hline
\end{tabular}




\section{Conclusions}

The EG prepared with $\mathrm{KMnO}_{4}$ as oxidant and $\mathrm{H}_{2} \mathrm{SO}_{4}$ as intercalator shows well catalytic activity in esterification of ethanol and malonnic acid under the following conditions: mole ratio of ethanol to malonnic acid is $6.0: 1$, reaction maintains slight boiling at about $72^{\circ} \mathrm{C}$, dosage of cyclohexane and the EG are $20 \mathrm{~mL}$ and $8 \mathrm{wt} \%$ respectively. Diethyl malonate yield can reach $77.6 \%$. The EG presents high stability, and it's easy to decentralize, separate and recycle.

Acknowledgments. The authors would like to thank Natural Science Foundation of Hebei Province (CN) (No. B2015201028) for financial support. Many thanks for Seedling Project of College of Chemistry and Environmental Science (Hebei University) for financial support.

\section{References}

1. S. Akbar and K. Srinivasan, "Iodine-catalyzed synthesis of highly functionalized 1h-indene derivatives from michael adducts of o-alkynylarene chalcones with diethyl malonate," European Journal of Organic Chemistry, vol. 2015, no. 35, pp. 7652-7655, 2015.

2. T. Kasumov and H. Brunengraber, "An improved procedure for the synthesis of labelled fatty acids utilizing diethyl malonate," Journal of Labelled Compounds and Radiopharmaceuticals, vol. 49, no. 2, pp. 171-176, 2006.

3. P. Shah, V. Ramaswamy, A. V. Ramaswamy and K, Lazar, "Synthesis and characterization of tin oxide-modified mesoporous SB A-15 molecular sieves and catalytic activity in trans-esterification reaction," Applied Catalysis A: General, vol. 273, no. 1, pp. 239-248, 2004.

4. H. Sachsse, "Ullmanns encyklopädie der technischen chemie, herausgeg. von w. foerst. band 3-10. verlag urban \& schwarzenberg, münchen-berlin 1953/58. 3. aufl., geb. dm 128. - pro band," Angewandte Chemie, vol. 72, no. 7-8, pp. 283-284, 1960.

5. W. H. Song and X. Z. Jiang, "A novel synthesis of diethyl malonate," Chinese Chemical Letters, vol. 11, no. 12, pp. 1035-1036, 2000.

6. T. Parangi, B. Wani and U. Chudasama, "Synthesis of monoesters and diesters using eco-friendly solid acid catalysts - Cerium(IV) and thorium(IV) phosphates," Applied Catalysis A: General, vol. 467, pp. 430-438, 2013.

7. B. Deng, A. H. Zhang and A. W. Xu, "Synthesis of diethyl malonate catalyzed by chitosan sulfate," China Surfactant Detergent \& Cosmetics, vol. 38, no. 5, pp. 313-315, 2008.

8. H. Shioyam and R. Fujii, "Electrochemical reactions of stage 1 sulfuric acid-Graphite intercalation compound," Carbon, vol. 25, no. 6, pp. 771-774, 1987.

9. J. P. Liu and K. M. Song, "The preparation of expandable graphite," Function Material, vol. 29, no. 6, pp. 659661, 1998.

10. Y. Q. Zhang, C. Wang, J. J. Ma, Y. H. Gao, Q. F. Geng, R. X. Tang and Y. J. Gao, "Synthesis of 3,3,6,6tetramethyl-9-aryl-1,2,3,4,5,6,7,8-xanthene- 1,8-dione catalyzed by expansible graphite," Chinese Journal of Organic Chemistry, vol. 27, no. 9, pp. 1147-1149, 2007.

11. X. Y. Pang, "Preparation of expandable graphite loaded with zinc sulfate and its catalytic activity in the synthesis of diisoamyl oxalate," International Journal of ChemTech Research, vol. 6, no. 5, pp. 3146-3151, 2014.

12. X. Y. Pang, "Catalytic behaviors of expansible graphite in the synthesis of butyl acetate," E Journal of Chemistry, vol. 9, no. 4, pp. 1816-1822, 2012.

13. J. J. Shao, "Direct synthesis and thermo dynamics calculation of malonicester," Journal of Heilongjiang Institute of Science \& Technology, vol. 18, no. 4, pp. 272-275, 2008.

14. X. Y. Pang, Z, Y, Dong and X. L. Li, "Study on some technology problems in the preparation of expandable graphite with fine flake graphite by chemical oxidation method," Chinese Carbon, no. 2, pp. 35-37, 2007.

15. M. Inagaki, T. Nagata, T. Suwa and M. Toyoda, "Sorption kinetics of various oils onto exfoliated graphite," New Carbon Materials, vol. 21, no. 2, pp. 97-102, 2006.

16. C. Q. Wang, F. Y. Ge, J. Sun and Z. S. Cai, "Effects of expandable graphite and dimethyl methylphosphonate on mechanical, thermal, and flame-retardant properties of flexible polyurethane foams," Journal of Applied Polymer Science, vol. 130, no. 2, pp. 916-926, 2013. 